\title{
Estoque domiciliar de medicamentos de pessoas assistidas por uma equipe de profissionais da Estratégia de Saúde da Família
}

\author{
Medications stored at home by patients cared for by Family Health Strategy professionals \\ Almacenamiento medicamentos de los pacients atendidos por profesionales de atención \\ primaria de la salud
}

Ana Paula Loch. Universidade Regional de Blumenau (FURB). Blumenau, SC, Brasil. anapaulaloch@gmail.com (Autora correspondente)

Nevoni Goretti Damo. Universidade Regional de Blumenau (FURB). Blumenau, SC, Brasil. nevoni@furb.br

Ernani Tiaraju de Santa Helena. Universidade Regional de Blumenau (FURB). Blumenau, SC, Brasil. etshelena@gmail.com

Edson Machado Sirai Missugiro. Santa Casa de Ribeirão Preto. Ribeirão Preto, SP, Basil. emsmitsu@ gmail.com

\section{Resumo}

Objetivo: identificar aspectos relacionados às "Farmácias caseiras" de pessoas assistidas por uma equipe da Estratégia Saúde da Família (ESF). Métodos: estudo transversal em 100 domicílios em uma área de abrangência da Estratégia Saúde da Família em Blumenau, SC, entre fevereiro e março de 2012. Resultados: nos domicílios visitados, 91\% tinham medicamentos, sendo encontrados 737 medicamentos (média de 7,6 por domicílio). $O$ grupo terapêutico de medicamentos mais frequente foi o dos que atuam no sistema digestivo $148(20,1 \%)$ e o medicamento mais encontrado foi o paracetamol $(6,5 \%)$. Dentre os entrevistados, 29 (31,9\%) relataram descartar medicamentos no lixo comum, 12 $(13,2 \%)$ não observavam o aspecto físico antes de utilizar e $74(81,3 \%)$ tiveram acesso a medicamentos em farmácias comunitárias e ESFs. Observou-se associação entre número de medicamentos e número de pessoas no domicílio $(p<0,01)$ e com presença de doenças crônicas $(p<0,05)$. Conclusão: pessoas com mais escolaridade armazenam mais medicamentos $(p<0,001)$. Contudo, pessoas com baixa escolaridade utilizam mais medicamentos prescritos por médicos $(p<0,001)$.

\begin{abstract}
Objective: to identify related aspects such as "home pharmacy" of people assisted by one of the Family Health Strategy (FHS). Methods: a cross-sectional study of 100 households in an area covered under the Family Health Strategy was conducted in Blumenau, SC, between February and March 2012. Results: among the households visited, 91\% had stored drugs; 737 different medications were found (with a mean of 7.6 medications per household). The most commonly stored drugs were the ones that act on the digestive system ( 148 drugs, or $20.1 \%$ ), and the most commonly found medication was paracetamol (6.5\%). Among the respondents, $29(31.9 \%)$ reported often having disposed of medications in the trash; 12 (13.2\%) did not particularly note the drug's appearance before ingesting it; and 74 (81.3\%) had access to drugs in publicly funded (community) pharmacies and primary healthcare centers. The number of drugs was associated with the number of people in the household $(p<0.01)$ and the presence of chronic diseases $(p<0.05)$. Conclusion: people with higher levels of education stored more drugs $(p<0.001)$. However, people with lower levels of education used more drugs prescribed by doctors $(p<0.001)$.
\end{abstract}

Como citar: Loch AP, Damo NG, Helena ETS Missugiro EMS. Estoque domiciliar de medicamentos de pessoas assistidas por uma equipe de profissionais da Estratégia de Saúde da Família. Rev Bras Med Fam Comunidade. 2015;10(37)1-11.

http://dx.doi.org/10.5712/rbmfc10(37)1090
Palavras-chave:

Armazenamento de

Medicamentos

Medicamentos

Uso de Medicamentos

Autocuidado

Atenção Farmacêutica

Keywords:

Drug Storage

Medications

Drug Utilization

Self Care

Pharmaceutical Care

Fonte de financiamento: Programa de Educação para o Trabalho em Saúde (PET-SAÚDE).

Parecer CEP:

FURB, 196/11, 09/02/2012.

Conflito de interesses: declaram não haver.

Procedência e revisão por pares: revisado por pares.

Recebido em: 14/03/2015.

Aprovado em: 21/11/2015. 


\section{Resumen}

Objetivo: identificar los aspectos relacionados con las "farmacias de casa" de personas atendidas por un equipo de la Estrategia Salud de la Familia (ESF). Métodos: un estudio transversal se llevó a cabo en 100 hogares en un área de cobertura de la Estrategia de la Salud de la Familia en Blumenau, SC, entre febrero y marzo del 2012. Resultados: en el $91 \%$ de los hogares visitados había medicinas, donde fueron encontrados 737 medicamentos (promedio de 7,6 por hogar). Los medicamentos que actúan sobre el sistema digestivo fue el grupo terapéutico más frecuente 148 (20,1\%) y la medicina más encontrada fue el Paracetamol (6,5\%). Entre los encuestados, $29(31,9 \%)$ relataron que decartaan sus medicamentos en la basura comun, $12(13,2 \%)$ no observaban el aspecto físico antes del uso y $74(81,3 \%)$ tuvieron acceso a los medicamentos en las farmacias comunitarias y en la ESF. Se observó una asociación entre el número de medicamentos y el número de personas en el hogar $(p<0,01)$ y la presencia de enfermedades crónicas $(p<0,05)$. Conclusión: las personas con más educación almacenan más drogas $(p<0,001)$. Sin embargo las personas con bajo nivel de estudios útilizan nás medicamentos prescritos por los médicos $(p<0,001)$.
Palabras clave:

Almacenamiento de

Medicamentos

Medicamentos

Utilización de Medicamentos

Autocuidado

Atención Farmacéutica

\section{Introdução}

O envelhecimento populacional e o aumento da expectativa de vida trazem consigo o aumento das doenças crônico-degenerativas, que gera novas demandas de consumo de serviços de saúde pela população. $\mathrm{O}$ consumo de medicamentos de modo contínuo está relacionado diretamente a essas necessidades de saúde. ${ }^{1}$

De acordo com Bueno et al., ${ }^{2}$ é muito frequente a população armazenar medicamentos e outros insumos relacionados à saúde nos domicílios, estocando-os de forma a constituir um estoque domiciliar, denominando este armazenamento como "farmácia caseira", independente do local que está localizada e das condições que se encontra.

Schenkel et al. ${ }^{3}$ consideram que o estoque domiciliar é composto por todos os medicamentos que estiverem sendo mantidos no domicílio e relatam ainda que existe uma escassez de dados de como os medicamentos são armazenados e utilizados após a sua aquisição. A forma de se verificar o que acontece com os medicamentos é avaliar a farmácia caseira, buscando respostas para o que acontece quando o medicamento chega às mãos do paciente.

Ribeiro e Heineck ${ }^{4}$ enfatizam que o excesso de medicamentos nos domicílios pode influenciar os moradores a praticar a automedicação e ainda gerar um problema de aquisição de medicamentos pelo SUS, pois se houver um programa eficiente de controle da entrega dos medicamentos, o ciclo da assistência farmacêutica se torna mais efetivo frente à escassez de recursos enfrentada nos dias de hoje. Achados semelhantes foram obtidos por Tourinho et al., ${ }^{5}$ que observaram que a automedicação entre crianças e adolescentes se mostra associado a uso de muitos medicamentos e a baixa escolaridade dos pais.

Desta forma, o objetivo desse estudo foi identificar aspectos relacionados às "Farmácias caseiras" de pessoas assistidas por uma equipe da Estratégia Saúde da Família (ESF).

\section{Método}

\section{Tipo de estudo}

Trata-se de um estudo epidemiológico descritivo transversal, realizado entre os meses de fevereiro e março de 2012, na área de abrangência de uma ESF do município de Blumenau/SC, cuja unidade de estudo foi o domicílio. Essa unidade de saúde é considerada docente-assistencial, fazendo parte de cenário de 
práticas para acadêmicos da Universidade Regional de Blumenau (FURB), dos cursos da área da saúde, além de participar do Programa de Educação para o Trabalho em Saúde (PET-SAÚDE).

\section{Seleção da amostra}

A equipe de saúde é composta por um médico, uma enfermeira, duas auxiliares de enfermagem e três agentes comunitárias de saúde (ACS), atende 841 famílias cadastradas distribuídas em 5 microáreas. Dos 841 domicílios cadastrados na unidade, foi escolhida uma amostra de 100 domicílios para serem visitados. Os domicílios foram selecionados tendo em conta a rotina dos ACS que inclui a entrega de receitas médicas, preenchimento de fichas A, agendamento de exames e consultas, marcação de puericultura e convocação para alguns usuários participantes de grupos. Algumas visitas foram realizadas somente pela pesquisadora e se limitaram a locais mais próximos da unidade de saúde. Houve uma distribuição heterogênea da amostra entre as microáreas, as microáreas 01 e 02 localizadas mais próximas à unidade de saúde concentraram 55\% da amostra, enquanto as microáreas 03, 04 e 05 representaram 45\%. Foram excluídos da amostra inicial $5 \%$ dos domicílios, $4 \%$ porque os moradores estavam ausentes, e $1 \%$ por recusa a participar. Estes domicílios foram substituídos por outros da mesma microárea.

\section{Roteiro de visita usado nos domicílios}

As entrevistas se sucederam ao longo do tempo, conforme o aceite dos moradores. Os domicílios foram visitados uma única vez pela entrevistadora.

Ao chegar aos domicílios, era explicado ao morador o objetivo do estudo e o mesmo era convidado a participar. Após a assinatura do Termo de Consentimento Livre e Esclarecido (TCLE), o morador era entrevistado com base em questionário estruturado adaptado (Bueno et al.) ${ }^{2}$ e era realizada uma análise visual dos medicamentos presentes nos domicílios e registro escrito.

\section{Variáveis do estudo}

No questionário utilizado na entrevista, foram abordadas as seguintes variáveis de estudo: idade, sexo, escolaridade (classificada em sete grupos: analfabeto, $1^{\circ}$ grau incompleto, $1^{\circ}$ grau completo, $2^{\circ}$ grau incompleto, $2^{\circ}$ grau completo, $3^{\circ}$ grau incompleto e $3^{\circ}$ grau completo), número de pessoas que residem no domicílio, classificando-as em crianças, adultos e idosos, presença de doença crônica, se sim, qual doença e qual o medicamento era utilizado para a mesma, quem havia prescrito/indicado os medicamentos, o local de aquisição dos medicamentos, se o entrevistado analisava o aspecto do mesmo antes de utilizá-lo, o local de armazenamentos no domicílio, se o medicamento estava ao alcance de crianças, presença de bula com o respectivo medicamento, local de descarte e prática de automedicação.

\section{Análise}

Somente os medicamentos de uso em humanos foram considerados como integrantes da farmácia caseira (excluindo preparações de uso animal). Os medicamentos foram agrupados com base no sistema de classificação Anatomical Therapeutic Chemical (ATC) do Nordic Council on Medicines, index 2012 (World Health Organization). ${ }^{6}$ O registro de medicamentos da Agência Nacional de Vigilância Sanitária 
(ANVISA) foi utilizado para identificar a composição das especialidades farmacêuticas. ${ }^{7}$ Foi examinado se os medicamentos constavam na Relação Nacional de Medicamentos Essenciais (RENAME) ${ }^{8}$ e na Relação Municipal de Medicamentos Essenciais (REMUME). ${ }^{9}$

Os dados foram digitados no aplicativo Epidata. Foram realizadas estatísticas descritivas apresentadas por frequência absoluta e frequência relativa com intervalo de $95 \%$ de confiança. Foi examinada a possível associação entre o estoque domiciliar (número de medicamentos, guarda e descarte) e características dos moradores (quantidade, sexo, idade, escolaridade e doenças). Para variáveis quantitativas, foram calculadas as medidas de tendência central (média e mediana) e de dispersão (desvio padrão). Foram utilizados os testes de " $t$ de Student" para comparar possíveis diferenças entre valores médios de variáveis contínuas e teste de Qui-Quadrado $\left(\chi^{2}\right)$ para associação entre variáveis categóricas. Foi aceito um nível de significância de $p<0,05$.

\section{Aspectos éticos}

O estudo foi feito em conformidade com a Declaração de Helsinque e aprovado pelo Comitê de Ética em Pesquisa da Universidade Regional de Blumenau (FURB), de acordo com o parecer constante do processo de número 196/2011.

\section{Resultados}

Foram visitados 100 domicílios nos quais uma pessoa consentiu em participar do estudo. O número de pessoas por domicílio variou de uma a nove pessoas (média=3,3 pessoas, $\mathrm{dp}=1,6$; mediana=3, intervalo interquartílico=2-4). A composição domiciliar foi predominantemente de adultos (somente $25 \%$ dos domicílios tinham crianças e $10 \%$ tinham idosos).

Os entrevistados tinham em média 42,5 anos ( $d p=15,7)$ e mediana de 41,6 anos (intervalo interquartílico 29,1-54,6), $77 \%$ eram do sexo feminino e $58,8 \%$ estudaram até o ensino fundamental (Tabela 1).

Tabela 1. Características sociodemográficas dos entrevistados.

\begin{tabular}{|c|c|c|}
\hline Variável & Frequência & Percentual \\
\hline \multicolumn{3}{|l|}{ Sexo $(n=100)$} \\
\hline Masculino & 27 & 27 \\
\hline Feminino & 73 & 73 \\
\hline \multicolumn{3}{|l|}{ Idade em anos $(n=100)$} \\
\hline Até 20 & 7 & 7 \\
\hline $20-49$ & 59 & 59 \\
\hline $50-64$ & 26 & 26 \\
\hline 65 e mais & 8 & 8 \\
\hline \multicolumn{3}{|l|}{ Escolaridade $(\mathrm{n}=97)$} \\
\hline Analfabeto & 2 & 2,1 \\
\hline Ensino fundamental incompleto & 36 & 37,1 \\
\hline Ensino fundamental completo & 19 & 19,6 \\
\hline Ensino médio incompleto & 6 & 6,2 \\
\hline Ensino médio completo & 23 & 23,7 \\
\hline Ensino superior incompleto & 8 & 8,2 \\
\hline Ensino superior completo & 3 & 3,1 \\
\hline
\end{tabular}


Com relação aos domicílios, 91\% possuíam medicamentos constituindo farmácia caseira, que se mostrou associado à presença de pessoas com doenças crônicas (todos os domicílios que tinham pessoas com essa condição possuíam farmácia caseira), mas não com o número de moradores. Dentre as características dos entrevistados, a presença de farmácia caseira se mostrou associada ao sexo feminino $\left(97,3 \% \times 74,1 \%, \chi^{2}=12,9 p<0,001\right)$.

Nas farmácias caseiras foram identificadas 737 especialidades farmacêuticas, que representa uma média de 7,6 por domicílio ( $d p=6,6)$ e mediana de 6 (intervalo interquartílico=3-10) e 2,2 por morador. Os dez medicamentos mais frequentes representam $32 \%$ do total, dos quais todos constam na REMUME e dois não constam na RENAME (Diclofenaco de potássio e Butilbromento de escopolamina) (Tabela 2).

Tabela 2. Especialidades farmacêuticas encontradas com maior frequência nos domicílios, segundo código anatômico, terapêutico e químico e ordem de frequência da especialidade.

\begin{tabular}{lcccc}
\hline ATC* $^{*}$ & Especialidade Farmacêutica & Frequência & $\%$ & IC95\% \\
\hline N02BE01 & Paracetamol & 48 & 6,5 & $4,8-8,5$ \\
M01AX17 & Nimesulida & 28 & 3,8 & $2,5-5,4$ \\
A02BC01 & Omeprazol & 26 & 3,5 & $2,3-5,1$ \\
M01AE01 & Ibuprofeno & 24 & 3,4 & $2,1-4,8$ \\
B01AC06 & AAS & 20 & 2,7 & $1,7-4,2$ \\
N02BB02 & Dipirona & 20 & 2,7 & $1,7-4,2$ \\
C10AA01 & Sinvastatina & 17 & 2,3 & $1,4-3,7$ \\
C09AA02 & Enalapril & 16 & 2,2 & $1,2-3,5$ \\
M01AB05 & Diclofenaco potássico & 15 & 2 & $1,1-3,3$ \\
A03BB01 & Butilbrometo de escopolamina & 14 & 1,9 & $1,0-3,2$ \\
Outros ${ }^{* *}$ & - & 527 & 68,9 & 100 \\
Total & & 737 & & \\
\hline
\end{tabular}

* Anatomic therapeutic chemical code; ** Correspondem a medicamentos com frequência inferior a 1,9\%.

Em relação à classificação anatômica das especialidades encontradas, as mais frequentes correspondem ao aparelho digestivo e metabolismo 149 (20,22\%), sistema nervoso 145 (19,67\%) e sistema musculoesquelético $115(15,60 \%)$. As classes terapêuticas que apresentaram maior frequência estão disponíveis na Tabela 3.

Tabela 3. Classes terapêuticas mais frequentes nos domicílios.

\begin{tabular}{clcc}
\hline ATC 2 o Nível & Classe Terapêutica & N & \% \\
\hline A01 & Preparações estomacais & 5 & 0,68 \\
A02 & Antiácidos & 41 & 5,56 \\
A03 & Fármacos para disfunções gastrointestinais funcionais & 52 & 2 \\
A04 & Antieméticos/antinauseantes & 3 & 0,27 \\
A05 & Terapia para bile e fígado & 10 & 0,41 \\
A06 & Laxativos & 11 & 1,36 \\
A07 & Antidiarreicos/Anti-inflamatórios intestinais/Anti-infecciosos intestinais & 11 & 1,49 \\
A10 & Drogas usadas no diabetes & 6 & 1,49 \\
A11 & Vitaminas & 3 & 0,81 \\
A12 & Suplementos minerais & 3 & 0,41 \\
A16 & Outras drogas para trato digestivo e metabolismo & 4 & 0,54
\end{tabular}


Tabela 3. Continuação...

\begin{tabular}{|c|c|c|c|}
\hline B01 & Antitrombóticos & 19 & 2,58 \\
\hline B03 & Antianêmicos & 10 & 1,36 \\
\hline $\mathrm{C} 01$ & Terapia cardíaca & 4 & 0,54 \\
\hline $\mathrm{CO3}$ & Diuréticos & 17 & 2,31 \\
\hline $\mathrm{C05}$ & Vasoprotetores & 8 & 1,09 \\
\hline C09 & Agentes que atuam no sistema renina-angiotensina & 26 & 3,53 \\
\hline C10 & Antidislipidêmicos & 28 & 3,80 \\
\hline D01 & Antifúngicos & 7 & 0,95 \\
\hline D02 & Emolientes & 4 & 0,54 \\
\hline D08 & Antissépticos/desinfetantes & 6 & 0,81 \\
\hline G03 & Hormônios sexuais e moduladores do sistema genital & 10 & 1,36 \\
\hline G04 & Urológicos & 4 & 0,54 \\
\hline $\mathrm{H} 02$ & Corticosteroides de uso sistêmico & 10 & 1,36 \\
\hline $\mathrm{HO3}$ & Terapia tireoide & 9 & 1,22 \\
\hline $\mathrm{J} 01$ & Antibacterianos de uso sistêmico & 26 & 3,53 \\
\hline M01 & Anti-inflamatórios e antirreumáticos & 106 & 14,38 \\
\hline M02 & Anti-inflamatório não esteroidais de uso tópico & 3 & 0,41 \\
\hline M03 & Relaxante muscular & 4 & 0,54 \\
\hline R01 & Preparações nasais & 14 & 1,90 \\
\hline R03 & Drogas para doenças obstrutivas das vias respiratórias & 14 & 1,90 \\
\hline R05 & Preparações para tosse e resfriado & 16 & 2,17 \\
\hline R06 & Antihistamínicos de uso sistêmico & 26 & 3,53 \\
\hline S01 & Oftalmológicos & 8 & 1,09 \\
\hline \multirow[t]{2}{*}{ S02 } & Otológicos & 3 & 0,41 \\
\hline & Outros* & 13 & 1,76 \\
\hline Total & & 737 & 100,00 \\
\hline
\end{tabular}

${ }^{*}$ Correspondem a medicamentos com frequência absoluta inferior a $\mathrm{n}=1$.

Chama a atenção que, apesar de os antibióticos representarem somente $3,5 \%$ do total de medicamentos prescritos, os mesmos estavam presentes em 21 dos 91 domicílios $(23,1 \%)$ que possuíam farmácia caseira.

Quando perguntados sobre o local de armazenamento dos medicamentos, $73(75,25 \%)$ citaram armazenar em algum local da cozinha, sendo: 41 (42,26\%) no armário superior, 14 (14,43\%) no balcão da cozinha, $8(8,24 \%)$ no armário inferior, $5(5,15 \%)$ sobre a geladeira, $3(3,09 \%)$ dentro da geladeira, enquanto 
$1(1,03 \%)$ armazenava sobre a mesa da cozinha ou em uma sacola plástica pendurada na parede. Outros sete $(14,43 \%)$ em algum lugar do dormitório e outros dois $(10,3 \%)$ em outros locais.

Quanto ao descarte, 29 (31.9\%) relataram descartar no lixo comum, 12 (13.2\%) afirmaram não observar o aspecto físico do medicamento antes de utilizar e $74(81,3 \%)$ relataram ter acesso a medicamentos em farmácias comunitárias e ESFs.

Observou-se que $64 \%$ dos domicílios com três ou mais pessoas tinham mais de dez medicamentos enquanto $59 \%$ dos domicílios com menos de três pessoas tinham até três medicamentos $\left(\chi^{2}=25,9\right.$ $\mathrm{p}<0,001)$. Por outro lado, $65,8 \%$ dos domicílios que tinham pessoas com doenças crônicas tinham mais de dez medicamentos enquanto $46,3 \%$ dos domicílios sem pessoas com doenças crônicas tinham até 3 medicamentos $\left(\chi^{2}=8,8 p<0,05\right)$.

Outras associações observadas se referem à escolaridade dos entrevistados. Pessoas com ensino médio ou superior armazenavam mais de dez medicamentos no domicílio $\left(59,8 \% \times 40,2 \%, \chi^{2}=15,2 p<0,01\right)$, mas com a presença de bula junto com os mesmos $\left(71,6 \% \times 28,4 \%, \chi^{2}=20,0 p<0,001\right)$. Por outro lado, pessoas com baixa escolaridade (analfabetos e com ensino fundamental) armazenam mais medicamentos ao alcance de crianças $\left(68,8 \% \times 31,2, \chi^{2}=13,4 p<0,001\right)$.

\section{Discussão}

Em 2001, foi implantada no Brasil a Política Nacional de Medicamentos (PNM), cujas diretrizes zelam pelo acesso a medicamentos de qualidade à população. ${ }^{1}$ Para tal objetivo, foram criadas algumas diretrizes, e entre elas está como prioritária a revisão permanente da Relação Nacional de Medicamentos Essenciais (RENAME), que permitiu que a população tenha acesso sobre prescrição médica a uma gama de medicamentos considerados essenciais, independente de fatores econômicos. Esta diretriz pode influenciar na formação de estoque domiciliar de medicamentos e consequente uso desses medicamentos estocados. Isso pode ter refletido nos resultados encontrados, pois como pode ser visto na Tabela 2, todas as especialidades que apresentaram maior frequência são as disponibilizadas na REMUME$^{9}$ do município de Blumenau e apenas três não estão disponíveis na RENAME. ${ }^{8}$

A prevalência de estoque domiciliar de medicamentos foi de $91 \%$, resultados semelhantes ao encontrado em estudos Brasileiros de diferentes regiões do país como os de Ferreira et al. ${ }^{10}$ em Divinópolis, MG, em que a presença de medicamentos nas residências foi de 96,6\% e Schenkel et al. ${ }^{3}$ em Porto Alegre, RS também observaram alto índice de medicamentos nas residências $97 \%$. Em outros países temos o estudo de Yousif ${ }^{11}$ em domicílios do Sudão, aproximadamente 97,7\% destes apresentaram pelo menos um medicamento e Nova Guiné, por Kiyingi \& Lauwo, ${ }^{12}$ das 139 famílias entrevistadas, 73 tinham o hábito de estocar medicamentos.

Nesse estudo, a média de especialidades farmacêuticas por domicílio foi de 7,6 especialidades, que se assemelha à encontrada no Rio Grande do Sul, ${ }^{13}$ cuja média foi de 7,5 por domicílio, enquanto na Bahia encontrou-se média de 9,2 por domicílio, ${ }^{14}$ o que sugere pouca variação em relação à média de especialidades farmacêuticas por domicílio nas diversas regiões brasileiras.

Algumas hipóteses podem ser formuladas para explicar esses resultados, que mostram o hábito de formar estoque domiciliar de medicamentos no Brasil. De um lado, aumento no acesso e a prática de automedicação, o crescente aumento de informações disponíveis sobre doenças e tratamentos e pela 
falta de controle nas vendas de medicamentos que exigem apresentação da receita médica e pela prática da prescrição inadequada. Por outro lado, aspectos relacionados ao modelo político-econômico da nossa sociedade que estimula o consumo, em que o medicamento é mais um produto a ser estocado. ${ }^{15}$ Apesar da RDC no 306 de 2004, ${ }^{16}$ que trata do gerenciamento de resíduos de serviços de saúde (RSS) - os RSS são de responsabilidade do próprio serviço que o gera, a falta de uma política de controle de resíduos por parte do Estado faz com que indústrias, distribuidoras e farmácias não se responsabilizem pelos seus resíduos. Diferentemente de países como Portugal, México, Canadá e Colômbia, o Brasil ainda não possui um programa nacional de recolhimento de medicamentos. ${ }^{17}$

A instauração de programas desse tipo associado a campanhas de conscientização dos usuários de medicamentos poderiam reduzir os estoques domiciliares. Outra medida importante nesse sentido é a dispensação de medicamentos fracionados conforme a necessidade do tratamento prescrito na receita médica, pelo serviço público e farmácias privadas. Vale lembrar que, no caso de tratamento de doenças crônicas, há a necessidade de um estoque domiciliar mínimo, para o qual o usuário deve contar com a orientação do profissional prescritor e do farmacêutico.

A maior proporção de entrevistados foi do sexo feminino, que pode ser atribuída ao fato de as mulheres terem maior disponibilidade para responder ao questionário e ainda permanecerem mais tempo no domicílio, sendo estas as integrantes da família que geralmente se responsabilizam pelos cuidados com saúde. ${ }^{18}$

Já o local predominante de armazenamento dos medicamentos foi a cozinha, o que se assemelha aos estudos. ${ }^{4,5,19,20}$ Tourinho et al. ${ }^{5}$ preconizam que o local utilizado para armazenar os medicamentos tem influência cultural e regional, sendo que em SP assim como em Minas Gerais, ${ }^{6}$ os autores verificaram que esse cômodo foi o "dormitório", que no presente estudo ficou em segundo lugar. Existe associação entre adesão medicamentosa e o local onde eles são armazenados, pois quanto mais práticos e próximos estão os medicamentos guardados, possivelmente maior será sua adesão. ${ }^{21}$

Foi observada uma associação entre a forma de armazenamento dos medicamentos e a escolaridade dos moradores. Pessoas com ensino médio ou superior armazenavam mais de dez medicamentos no domicílio, mas com a presença de bula junto, enquanto pessoas com baixa escolaridade (analfabetos e com ensino fundamental) armazenam mais medicamentos ao alcance de crianças.

Ao se tratar do armazenamento ao alcance das crianças, autores como Kusturica et al. ${ }^{15}$ mostram que as crianças são expostas a um alto risco acidental de intoxicações por medicamentos. Neste estudo, em 15 domicílios (16,48\%), os medicamentos encontravam-se ao alcance de crianças, resultado abaixo dos $22,40 \%$ encontrado no estudo por Tourinho et al. ${ }^{5}$ e $23,5 \%$ relatado por Milanez et al. ${ }^{18}$

Domicílios com pessoas com escolaridade menor possuem maior acesso aos medicamentos pelo Sistema Único de Saúde. Estudo de Rodrigues et al. ${ }^{21}$ já havia demonstrado que o uso dos serviços da Estratégia Saúde da Família está associado a pessoas de baixo nível escolar tanto no Nordeste quanto no Sul do Brasil. Nesse contexto, a presença de um profissional farmacêutico nas ESF poderia contribuir para minimizar tais erros de armazenamento por meio de orientações. Para facilitar o processo, o farmacêutico poderia contar com o auxílio das (os) profissionais ACSs para o diagnóstico de problemas de armazenamento e então realizar um atendimento individualizado para as pessoas com maiores dificuldades de entendimento devido à baixa escolaridade e outros possíveis fatores. 
Em relação às classes farmacológicas, os medicamentos com ação no aparelho digestivo e metabolismo foram os mais encontrados: 148 (20,08\%), seguidos do sistema nervoso 145 (19,67\%), músculo esquelético 115 (15,60), cardiovascular $114(15,47 \%)$ e respiratório 71 (9,63\%), resultados parecidos ao estudo de Arrais et al. ${ }^{22}$ e Ribeiro e Heineck, ${ }^{4}$ que encontraram os mesmos grupos de medicamentos.

$O$ índice de medicamentos encontrados relacionados ao sistema nervoso pode ser justificado, ao menos em parte, pelo uso como tratamento de apoio a stress pós-trauma, pois a maioria das casas e ruas no local do estudo é atingida por repetidas enchentes e enxurradas. Apesar da classificação anatômica do Sistema Nervoso englobar várias especialidades, incluindo medicamentos da classe dos anti-inflamatórios não esteroidais (AINES), aos quais a população tem fácil acesso em farmácias comunitárias, 18 (2,44\%) especialidades correspondiam à classe dos inibidores da recaptação de serotonina e $17(2,31 \%)$ à classe dos benzodiazepínicos.

Quanto aos medicamentos antimicrobianos, uma alteração da legislação № 44 , de $2010,{ }^{23}$ tornou obrigatória a dispensação de medicamentos antimicrobianos mediante prescrição médica. Neste estudo se encontraram 28 (3,80\%) antibióticos, enquanto no estudo realizado por Ribeiro e Heineck ${ }^{4}$ o número de antibióticos de uso sistêmico foi de 5,82\%. Por outro lado, apesar de os antibióticos representarem somente $3,5 \%$ do total de medicamentos prescritos, os mesmos estavam presentes em 21 dos 91 domicílios $(23,1 \%)$ que possuíam farmácia caseira, o que pode sugerir abandono parcial de tratamento com sobra de medicamentos que pode favorecer seu uso indevido que pode favorecer o fenômeno do aumento da resistência bacteriana. ${ }^{24}$

O vínculo do paciente com a sua medicação, como verificar as características físicas e organolépticas do medicamento antes de tomar, sugerem uma adesão maior ao tratamento. Sendo importante ressaltar que o local destinado ao armazenamento de medicamentos deve ser seco e ao abrigo da luz, no estudo apenas $12(13,19 \%)$ afirmaram não observar o aspecto físico do medicamento antes de utilizar, fato que pode ser reflexo da escolaridade da população estudada e baixo conhecimento sobre o assunto.

Já em relação ao acesso, $74(81,31 \%)$ relataram ter acesso a medicamentos em farmácias comunitárias e ESFs, fato que pode ocorrer devido à facilidade de aquisição dos medicamentos em farmácias comunitárias, visto que a maioria dos medicamentos podem ser adquiridos sem receita médica.

Esse estudo conta com algumas possíveis limitações. O processo amostral não foi constituído como uma amostra aleatória de todos os domicílios da área de abrangência da ESF o que pode gerar um viés de seleção. Contudo, os resultados se mostraram semelhantes a outros estudos nacionais no contexto da atenção primária quanto à composição dos domicílios e proporção das variáveis de estudo, o que sugere que esse viés, caso tenha ocorrido, não parece ter efeito importante. Por outro lado, como se trata de estudo seccional, não é possível estabelecer associações causais. Por fim, o fato se termos realizado o estudo em uma única ESF pode comprometer a generalização dos resultados.

\section{Conclusão}

Conclui-se que as farmácias caseiras são muito prevalentes nos usuários da atenção primária. Desta forma, fazem-se necessárias ações educativas das equipes de ESF junto à comunidade, no sentido de minimizar práticas irracionais de consumo, armazenamento e descarte de medicamentos, em especial para 
famílias numerosas e com pessoas com doenças crônicas. Outros estudos são necessários no sentido de melhor compreender os possíveis fatores associados à prática de estocar medicamentos, em especial no contexto da atenção primária.

\section{Referências}

1. Secretaria de Políticas de Saúde. Política nacional de medicamentos. Rev Saúde Pública. 2000;34(2):206-9. DOI: http:// dx.doi.org/10.1590/S0034-89102000000200018

2. Bueno CS, Weber D, Oliveira KR. Farmácia caseira e descarte de medicamentos no bairro Luiz Fogliatto do município de ljuí - RS. Rev Ciênc Farm Básica Apl. 2009;30(2):203-10.

3. Schenkel EP, Fernándes LC, Mengue SS. Como são armazenados os medicamentos nos domicílios? Acta Farm Bonaerense. 2005;24(2):266-70.

4. Ribeiro MA, Heineck I. Estoque Domiciliar de Medicamentos na Comunidade Ibiaense Acompanhada pelo Programa Saúde da Família, em Ibiá - MG, Brasil. Saúde Soc São Paulo. 2010;19(3):653-63.

5. Tourinho FSV, Bucaretchi F, Stephan C, Cordeiro R. Farmácias domiciliares e sua relação com a automedicação em crianças e adolescentes. J Pediatr (Rio J.). 2008;84(5):416- 22.

6. WHO - World Health Organization. WHO Collaborating Centre for Drug Statistics Methodology. The Anatomical Therapeutic Chemical Classification (ATC); 2014 [acesso 18 Nov 2015]. Disponível em: http://www.whocc.no/atc_ddd_index/

7. Agência Nacional de Vigilância Sanitária (ANVISA). Bulário Eletrônico; 2014 [acesso 18 Nov 2015]. Disponível em: http:// www.anvisa.gov.br/datavisa/fila_bula/index.asp

8. Brasil. Ministério da Saúde. Política Nacional de Medicamentos. Relação Nacional de Medicamentos Essenciais (RENAME). Brasília: Ministério da Saúde, 2013.

9. Prefeitura municipal de Blumenau, Secretaria Municipal da Saúde. Relação municipal de medicamentos. Blumenau, SC; 2012 [acesso 18 Nov 2015]. Disponível em: http://www.blumenau.sc.gov.br/downloads/semus/portaria_359.pdf

10. Ferreira WA, Silva MEST, Paula ACCFF, Resende CAMB. Avaliação de farmácia caseira no município de Divinópolis (mg) por estudantes do curso de farmácia da Unifenas. Infarma. 2005;17(7/9):84-6.

11. Yousif MA. In-home drug storage and utilization habits: a Sudanese study. East Mediterr Health J. 2002;8(2-3):422-31.

12. Kiyingi KS, Lauwo JA. Drugs in the home: danger and waste. World Health Forum. 1993;14(4):381-4. PMID: 8185788

13. Deitos A, Laste G, Marcolin F, Hammes L, Schwingel D, Weizanmann RS, et al. Análise do estoque domiciliar de medicamentos em municípios do vale do taquari - RS. XI Salão de Iniciação Científica - PUCRS, 09 a 12 de agosto de 2010 [acesso 18 Nov 2015]. Disponível em: http://www.pucrs.br/edipucrs/XISalaolC/Ciencias_da_Saude/Farmacia/82642ALICIA_DEITOS.pdf

14. Franco RCS, Carvalho Neto JA, Khouri MA, Nunes MO, Santos Júnior JW, Marques Neto J, et al. Consumo de medicamentos em um grupo populacional da área urbana de Salvador-BA. Rev Baiana Saúde Publica. 1987;13/14(4/1):113-21.

15. Kusturica MP, Sabo A, Tomic Z, Horvat O, SolakZ. Storage and disposal of unused medications: knowledge, behavior, and attitudes among Serbian people. Int J Clin Pharm, 34(4):604-10, 2012. DOI: http://dx. doi.org/10.1007/s1 1096-012-9652-0

16. Brasil. Ministério da Saúde. Agência Nacional de Vigilância Sanitária (ANVISA). RDC № 306 de 2004. Brasília: Ministério da Saúde; 2004.

17. Falqueto E, Kligerman DC. Diretrizes para um programa de recolhimento de medicamentos vencidos no Brasil. Ciênc Saúde Coletiva. 2013;18(3):883-92. DOl:http://dx.doi.org/10.1590/S1413-81232013000800034

18. Milanez MC, Stutz E, Rosales TO, Penteado AJ, Perez E, Cruciol e Souza JM, et al. Avaliação dos estoques domiciliares de medicamentos em uma cidade do Centro-Sul do Paraná. Rev Ciênc Méd Biol. 2013;12(3):283-9.

19. Shah AD, Wood DM, Dargan PI. Internet survey of home storage of paracetamol by individuals in the UK. QJM. 2013;106(3):253-9. Epub 2012 Dec 19. DOI: http://dx.doi.org/10.1093/qjmed/hcs232 
20. Krousel-Wood M, Islam T, Webber LS, Re RN, Morisky DE, Muntner P. New medication adherence scale versus pharmacy fill rates in seniors with hypertension. Am J Manag Care. 2009;15(1):59-66.

21. Rodrigues MA, Facchini LA, Piccini RX, Tomasi E, Thumé E, Silveira DS, et al. Use of primary care services by elderly people with chronic conditions, Brazil. Rev Saúde Pública. 2009;43(4):604-12. DOI: http://dx.doi.org/10.1590/S003489102009005000037

22. Arrais PSD, Brito LL, Barreto ML, Coelho HLL. Prevalência e fatores determinantes do consumo de medicamentos no Município de Fortaleza, Ceará, Brasil. Cad Saúde Pública. 2005;21(6):1737-46. DOI: http://dx.doi.org/10.1590/S0102$311 \times 2005000600021$

23. Brasil. Ministério da Saúde. Agência Nacional de Vigilância Sanitária (ANVISA). RDC № 44 de 2010. Brasília: Ministério da Saúde; 2010.

24. Brasil. Ministério da Saúde. Departamento de Assistência Farmacêutica e Insumos Estratégicos. Uso Racional de Medicamentos: temas selecionados. Brasília: Ministério da Saúde; 2012. 